\title{
Time evolution of three-dimensional nonlinear gravity-capillary free-surface flows
}

\author{
E. I. Părău · J. M. Vanden-Broeck • M. J. Cooker
}

Received: 29 December 2009 / Accepted: 9 July 2010 / Published online: 27 July 2010

(C) Springer Science+Business Media B.V. 2010

\begin{abstract}
The evolution in time of three-dimensional gravity and gravity-capillary free-surface flows generated by a moving pressure distribution is considered. Solutions of the fully nonlinear equations in deep water are calculated by boundary-integral-equation methods and marching in time. Comparisons between unsteady and steady solutions are discussed.
\end{abstract}

Keywords Boundary-integral method · Free-surface flows · Gravity-capillary waves ·

Steady and unsteady forced waves · Time-dependent water waves

\section{Introduction}

The study of gravity-capillary free-surface flows generated by disturbances moving at a constant velocity is a classical topic in fluid mechanics. Results have been obtained in both two and three dimensions. The disturbance can be a submerged object (e.g. a submarine), a surface-piercing object (e.g. a ship or an insect) or a prescribed distribution of pressure. The results presented in this paper are qualitatively independent of the type of disturbance and we shall restrict our attention to distributions of prescribed pressure.

Often steady solutions are sought in a frame of reference moving with the disturbance (see [1]). Analytical results can then be derived by assuming that the magnitude of the disturbance is small and by linearising the equations around a uniform stream. However, it is found that when waves are present, the solutions of the resulting linear equations are non-unique, unless a radiation condition is imposed. This condition requires that there is no energy coming from infinity. Rayleigh [2] showed that a convenient way to impose the radiation condition is to introduce some dissipation $\mu$ in the dynamic boundary condition, solve the problem and then take the limit as $\mu \rightarrow 0$. The dissipation coefficient $\mu$ is known as the Rayleigh viscosity.

Steady two- and three-dimensional fully nonlinear solutions have been computed by using boundary-integralequation methods [1,3-8]. One important question is how to impose the radiation condition in the nonlinear regime.

E. I. Părău $(\bowtie) \cdot$ M. J. Cooker

School of Mathematics, University of East Anglia, Norwich NR4 7TJ, UK

e-mail: e.parau@uea.ac.uk

J. M. Vanden-Broeck

Department of Mathematics, University College London, London WC1 E6BT, UK 
In the case of pure gravity flows (i.e., in the absence of surface tension), the radiation condition implies that the waves (if present) are behind the disturbance and that the free surface is essentially flat at some distance in front of the disturbance. This is relatively easy to impose numerically, by forcing the slope and/or the curvature of the free surface to be zero at the mesh points in front of the disturbance which are further away from the disturbance [1]. The case of gravity-capillary free-surface flows is much harder because there are now waves both at the front and at the back of the disturbance. The role of the radiation condition is then to select which of the waves are at the front and which are at the back of the disturbance. This is not easy to impose numerically, even in the two-dimensional case; see $[9,10]$ for calculations describing these difficulties and for ways to overcome them.

In previous work we developed a method to impose numerically the radiation condition for fully nonlinear threedimensional gravity-capillary free-surface flows [6]. The idea was to use in the fully nonlinear regime the technique used by Rayleigh for linear problems. In other words we solved numerically the nonlinear equations with a small Rayleigh viscosity $\mu$ included in the dynamic boundary condition. Solutions with $\mu \neq 0$ are then unique and satisfy the radiation condition. However, the trains of waves are damped because the Rayleigh viscosity $\mu$ is different from zero. It is therefore of interest to seek alternative methods that lead to solutions satisfying the radiation condition but without damping. In this paper we show that a way to achieve this goal is to solve the time-dependent problem. This is one of the motivations for the calculations presented in this paper.

Much progress on the numerical modelling of three-dimensional time-dependent free-surface flows has been achieved over the years. For a recent review, see [11]. In most of these previous studies the effect of surface tension was neglected. Here we develop an algorithm where the influence of surface tension is included in the dynamic boundary condition. It is based on a boundary-integral-equation method coupled with marching in time. For simplicity we restrict our attention to water of infinite depth. Our results extend previous studies in which gravity was included but the effect of surface tension neglected.

The formulation of the problem and the numerical procedure are described in Sect. 2. The results are presented in Sect. 3.

\section{Formulation and numerical scheme}

We consider a three-dimensional distribution of pressure moving at a constant velocity $U$ at the surface of a fluid of infinite depth. The fluid is assumed to be inviscid and incompressible and the flow to be irrotational. We choose a frame of reference moving with the pressure distribution. We introduce Cartesian coordinates $x, y, z$ with the $z$-axis directed vertically upwards and $z=0$ on the undisturbed level of the free surface. The $x$-axis is in the opposite direction of the velocity $U$ and we denote by $z=\zeta(x, y, t)$ the equation of the free surface. We use an Eulerian formulation in a frame of reference moving with the pressure distribution.

The velocity potential $\varphi(x, y, z, t)$ satisfies Laplace equation

$\nabla^{2} \varphi=0, \quad x, y \in \mathbb{R}, z<\zeta(x, y, t)$,

in the flow domain. At infinity we require the flow to approach a uniform flow with constant velocity $U$ in the $x$ direction by writing

$\varphi \rightarrow U x, \quad$ as $x^{2}+y^{2}+z^{2} \rightarrow \infty$.

We denote by $\Phi=\varphi-U x$ the perturbation potential, which also satisfies the Laplace equation (1) and decays to 0 at infinity in view of (2).

On the free surface, $z=\zeta(x, y, t)$, the full nonlinear kinematic condition and the dynamic boundary condition are applied. In the moving Eulerian frame of reference, these conditions are

$$
\begin{aligned}
& \zeta_{t}+U \zeta_{x}+(\mathbf{v} \cdot \nabla)(\zeta(x, y, t)-z)=0 \\
& \phi_{t}+U \phi_{x}+\frac{1}{2}(\mathbf{v} \cdot \mathbf{v})+g \zeta+\frac{p}{\rho}-\frac{T}{\rho}\left[\frac{\zeta_{x}}{\sqrt{1+\zeta_{x}^{2}+\zeta_{y}^{2}}}\right]_{x}-\frac{T}{\rho}\left[\frac{\zeta_{y}}{\sqrt{1+\zeta_{x}^{2}+\zeta_{y}^{2}}}\right]_{y}=0
\end{aligned}
$$


where $\phi(x, y, t)=\Phi(x, y, \zeta(x, y, t)), \mathbf{v}=\nabla \Phi$ is the fluid velocity, $T$ is the constant coefficient of surface tension, $g$ is the acceleration due to gravity and $\rho$ is the fluid density.

We introduce dimensionless variables by using $U$ as the unit velocity and $L$ (the length of the support of the pressure distribution) as the unit length.

We solve the problem by a boundary-integral-equation method coupled with marching in time. The boundaryintegral-equation method is similar to that used in [1], [4-8] for the steady case. Therefore we shall only describe it briefly and the reader is referred to these papers for further details.

We apply Green's theorem to $\Phi$ and the free-space Green function of Laplace's equation,

$G=\frac{1}{4 \pi} \frac{1}{\left(\left(x-x^{*}\right)^{2}+\left(y-y^{*}\right)^{2}+\left(z-z^{*}\right)^{2}\right)^{1 / 2}}$,

in a domain whose surface $S$ consists of a hemisphere of large radius, centred at the middle of the pressure disturbance, bounded by the free surface (except for a small hemisphere around the point $P^{*}\left(x^{*}, y^{*}, z^{*}\right)$ on the free surface $S_{F}$ ). Then we take the limit as this radius tends to infinity. We obtain

$-2 \pi \Phi\left(\mathbf{x}^{*}, t\right)=\int_{S}\left(\Phi(\mathbf{x}, t) \frac{\partial}{\partial n}\left(\frac{1}{\left|\mathbf{x}-\mathbf{x}^{*}\right|}\right)-\frac{\partial \Phi}{\partial n}(\mathbf{x}, t) \frac{1}{\left|\mathbf{x}-\mathbf{x}^{*}\right|}\right) \mathrm{d} S$,

where $\mathbf{x}=(x, y, z)$ and the point $\mathbf{x}^{*}=\left(x^{*}, y^{*}, z^{*}\right)$ is on the free surface. Here we use the unit outward normal at $P(x, y, z)$

$\mathbf{n}=\frac{\left(-\zeta_{x},-\zeta_{y}, 1\right)}{\left(\zeta_{x}^{2}+\zeta_{y}^{2}+1\right)^{1 / 2}}$

We treat the singularities in the integral (6) by following the analysis used in the steady case by Părău and Vanden-Broeck [1], Landweber and Macagno [12] and Forbes [3]. We add and subtract the null term

$\Phi\left(\mathbf{x}^{*}, t\right) \int_{S} \frac{\partial}{\partial n}\left(\frac{1}{\left|\mathbf{x}-\mathbf{x}^{*}\right|}\right) \mathrm{d} S$

to make the first integral in (6) non-singular, then we project it onto $\mathbb{R}^{2}$, the plane $O x y$, to obtain

$$
\begin{aligned}
-2 \pi \Phi\left(\mathbf{x}^{*}, t\right)= & \int_{\mathbf{R}^{2}}\left(\Phi(\mathbf{x}, t)-\Phi\left(\mathbf{x}^{*}, t\right)\right) \frac{\zeta_{x}\left(x-x^{*}\right)+\zeta_{y}\left(y-y^{*}\right)-\left(\zeta-\zeta^{*}\right)}{\left|\mathbf{x}-\mathbf{x}^{*}\right|^{3}} \mathrm{~d} x \mathrm{~d} y \\
& -\int_{\mathbf{R}^{2}} \frac{\left(\zeta_{x}^{2}+\zeta_{y}^{2}+1\right)^{1 / 2}}{\left|\mathbf{x}-\mathbf{x}^{*}\right|} \frac{\partial \Phi}{\partial n}(\mathbf{x}, t) \mathrm{d} x \mathrm{~d} y .
\end{aligned}
$$

At a given time we know $\Phi$ and $\zeta$. We substitute these values in (7) and solve for $\frac{\partial \Phi}{\partial n}(\mathbf{x}, t)$. We can then calculate $\zeta_{t}$ and $\phi_{t}$ from (3) and (4). The values of $\Phi$ and $\zeta$ at the next time step are then obtained by marching in time. Details are given below.

The discretization involves a regular grid with $n$ points in the $x$-direction, truncating $(-\infty, \infty)$ and $m$ points in the $y$-direction, truncating $(0, \infty)$. The uniform mesh sizes on the $x$-and $y$-axes are denoted by $\delta x$ and $\delta y$. It should be noted that the grid points move with the speed of the pressure distribution, so the pressure will always be centred at $(0,0)$.

The remaining singularity in the second term of the integral in (6) is removed as in the steady case, by adding and subtracting an appropriate quantity that can be evaluated in closed form; see $[1,3]$.

All the integrals are calculated by using the trapezoidal rule with a sum over the mesh points and are evaluated at the midpoints. Four-point finite-difference and interpolation formulae are used where needed.

The linear system to be solved by a direct method can be written in the form $A U=B$ with the notation

$U=\left(U_{1}, \ldots, U_{N}\right), \quad$ where $U_{I}=\frac{\partial \Phi}{\partial n}(i, j)$,

for $I=(i-1) m+j, i=1, \ldots, n, j=1, \ldots, m$ and $N=n m$. 
The boundary conditions (3)-(4) can be rewritten as

$$
\begin{aligned}
\zeta_{t}= & -\zeta_{x}+\Phi_{n}, \quad z=\zeta(x, y), \\
\phi_{t}= & -\phi_{x}-\frac{1}{2}\left[\left(\frac{\partial \Phi}{\partial n}\right)^{2}+\frac{\left(1+\zeta_{x}^{2}\right) \phi_{y}^{2}+\left(1+\zeta_{y}^{2}\right) \phi_{x}^{2}-2 \zeta_{x} \zeta_{y} \phi_{x} \phi_{y}}{1+\zeta_{x}^{2}+\zeta_{y}^{2}}\right] \\
& -\frac{\zeta}{F^{2}}-\varepsilon P+\beta\left[\frac{\zeta_{x}}{\sqrt{1+\zeta_{x}^{2}+\zeta_{y}^{2}}}\right]_{x}+\beta\left[\frac{\zeta_{y}}{\sqrt{1+\zeta_{x}^{2}+\zeta_{y}^{2}}}\right]_{y} .
\end{aligned}
$$

Here

$F=\frac{U}{\sqrt{g L}}$ and $\beta=\frac{T}{\rho U^{2} L}$.

Similar equations in a moving Eulerian frame of reference have been derived by other authors [13,14], but their work neglects the surface tension that we include here.

After computing $\frac{\partial \Phi}{\partial n}(\mathbf{x}, t)$, we can find the shape of the free surface and the velocity potential at the next time step by marching in time, i.e.,

$\zeta(x, t+\delta t) \approx \zeta(x, t)+\delta t \zeta_{t}(x, t), \quad \phi(x, t+\delta t) \approx \phi(x, t)+\delta t \phi_{t}(x, t)$.

To do so we use a predictor-corrector method, with an intermediary step. For some solutions the stepping in time was performed using the fourth-order Runge-Kutta method, to check the predictor-corrector method and very good agreement was found.

At each time step we also impose the following far-field boundary conditions at the first and last row in the $x$-direction and the last row in the $y$-directions

$\zeta_{1 j}=0, \quad \phi_{1 j}=0, \quad \zeta_{n, j}=2 \zeta_{n-1, j}-\zeta_{n-2, j}, \quad \phi_{n, j}=0, \quad \zeta_{i, m}=2 \zeta_{i, m-1}-\zeta_{i, m-2}, \quad \phi_{i m}=0$.

Here we use the notation $\zeta_{i j}=\zeta\left(x_{i}, y_{j}\right), \phi_{i j}=\phi\left(x_{i}, y_{j}\right), \zeta_{i j}=\zeta\left(x_{i}, y_{j}\right)$ etc.

The dimensionless pressure distribution which models the disturbance, is chosen as

$P(x, y)= \begin{cases}\mathrm{e}^{\frac{1}{x^{2}-1}+\frac{1}{y^{2}-1}}, & |x|<1 \text { and }|y|<1, \\ 0, & \text { otherwise. }\end{cases}$

Longuet-Higgins and Cokelet [15] found that in their two-dimensional problems, the free-surface profile may develop, after some time, a saw-tooth instability. A similar problem occurred in some of our computations and we then used the smoothing described in [15] to remove the instability. Also there are some disturbances which become visible at the upstream boundary, due to numerical errors accumulating there. Here we remove these disturbances by using a sponge or an absorbing beach on a narrow strip near the upstream boundary; see [11] and [16] for details.

\section{Results}

We used the scheme of Sect. 2 to compute solutions for various values of the parameters. We checked the accuracy of the results by varying the time step $\delta t$, and the spatial grid intervals $\delta x$ and $\delta y$.

We first validate our numerical approach by calculating solutions in the absence of surface tension. A typical free-surface profile for $F=0.7$ is shown in Fig. 1 for three different times. The Kelvin wake with the two wave systems of divergent and transverse waves is seen behind the pressure distribution. As $t \rightarrow \infty$, the solutions approach steady profiles. To confirm this, we compare in Fig. 2 a steady solution, computed using the algorithm described in Părău and Vanden-Broeck [1], with an unsteady solution calculated after a large number of time steps. A very good agreement is found between the divergent and the transverse wave systems. This demonstrates that steady solutions satisfying the radiation condition can be obtained as the long time behaviour of time-dependent solutions. There is 


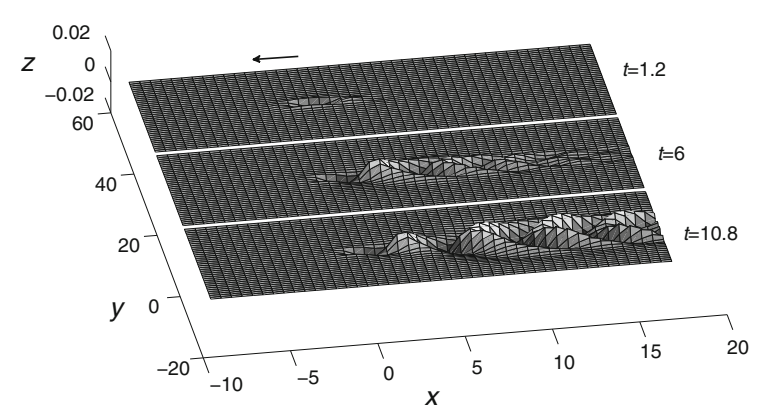

Fig. 1 Time-dependent forced gravity waves. In this computation we have used $n=60, m=20, \delta x=\delta y=0.6, \delta t=0.1$. The magnitude of the pressure is $\varepsilon=0.1$

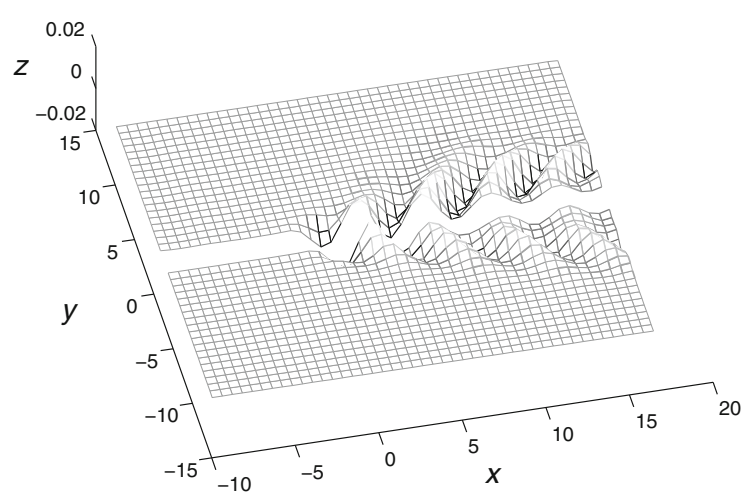

Fig. 2 Time-dependent and steady forced gravity waves for $F=0.7$. In the upper part of the figure $(y>0)$ we plotted the time-dependent solution at the time $t=10.8$. In this computation we have used $n=60, m=20, \delta x=\delta y=0.6, \delta t=0.1$. In the lower part of the figure $(y<0)$ we plotted the steady solution

a slight difference in the amplitudes of the solutions, with the time-dependent algorithm overpredicting them. This could be improved by decreasing $\delta x$ and $\delta y$.

Next we present new results when both the effects of gravity and surface tension are included in the dynamic boundary condition.

We introduce our time-dependent solutions by recalling the basic properties of steady solutions; see $[4,17,18]$ for more details. We introduce the parameter

$\alpha=\frac{\beta}{F^{2}}$.

It can be shown that linear waves can only occur on the free surface when $\alpha<1 / 4$. It is then necessary to impose the radiation condition to calculate steady solutions. As explained in the introduction, this can be achieved by introducing a small Rayleigh viscosity $\mu$.

When $\alpha>1 / 4$, there are no waves and the free-surface profiles are symmetric in $x$ with respect to $x=0$ (for distributions of pressure having the same symmetry). No radiation condition needs to be imposed in that regime. For the two-dimensional theory, see [17], for numerical computations, [19, pp. 457-468], for early results and [18] for a review of the subject. Numerical results in the three-dimensional case are presented in [4] and [5]. We shall refer to the range $\alpha>1 / 4$ as the regime of strong surface tension.

We first describe our results for time-dependent gravity-capillary solutions in a region of strong surface tension (i.e., for $\alpha>1 / 4$ ). In Fig. 3, solutions are shown for $F=1$ and $\beta=2$. Then $\alpha=2>1 / 4$ (see (11)). We present the time-dependent solutions at three different times and we observe that a steady depression begin to forms below the support of the pressure. There is another unsteady wave in front of the pressure distribution which travels faster than the pressure distribution.

We compare in Fig. 4 a steady gravity-capillary solution computed using the algorithm described in [5], with a time-dependent gravity-capillary wave solution obtained at $t=10.8$. It can be observed that, neglecting the unsteady wave which has detached from the pressure distribution, the solutions near the support of the pressure distribution are very similar. This is confirmed further by drawing the centrelines of these time-dependent solutions at various times and of the steady solution (see Fig. 5).

Our numerical scheme can be used to investigate the effect of varying the parameters $\beta$ and $F$. For example in Figs. 6 and 7 three solutions are plotted at the same time but for different values of $F$. Here $\beta=2$. As $F$ increases, we observe that the depth of the depression under the pressure distribution increases. The time-dependent waves 


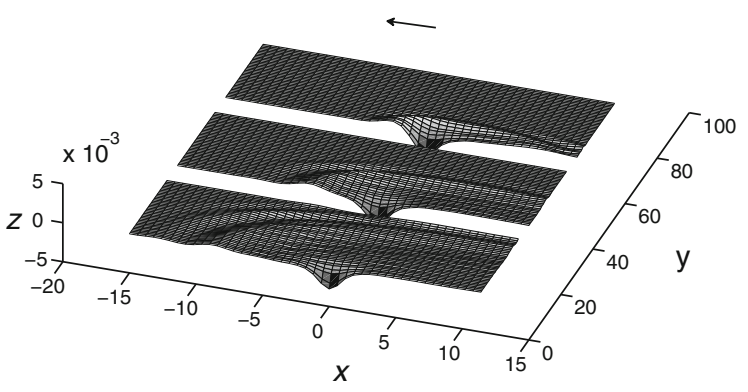

Fig. 3 Time-dependent forced gravity-capillary waves for $\beta=2, F=1$ at different times $t=2.4,6$ and 10.8 (top/middle/bottom). In this computation we have used $n=60, m=$ $40, \delta x=\delta y=0.6, \delta t=0.1$. Only half of the solutions $(y>0)$ are plotted

Fig. 5 Centreline $z=\zeta(x, 0)$ of the time-dependent forced gravity-capillary waves at different times (solid lines) and steady forced gravity-capillary waves (dashed line) for $\beta=2, F=1$



Fig. 4 Time-dependent forced gravity-capillary waves at $t=$ 10.8 and steady forced gravity-capillary waves (lower part of the graph) for $\beta=2, F=1$. Only half of the solutions $(y>0)$ are plotted

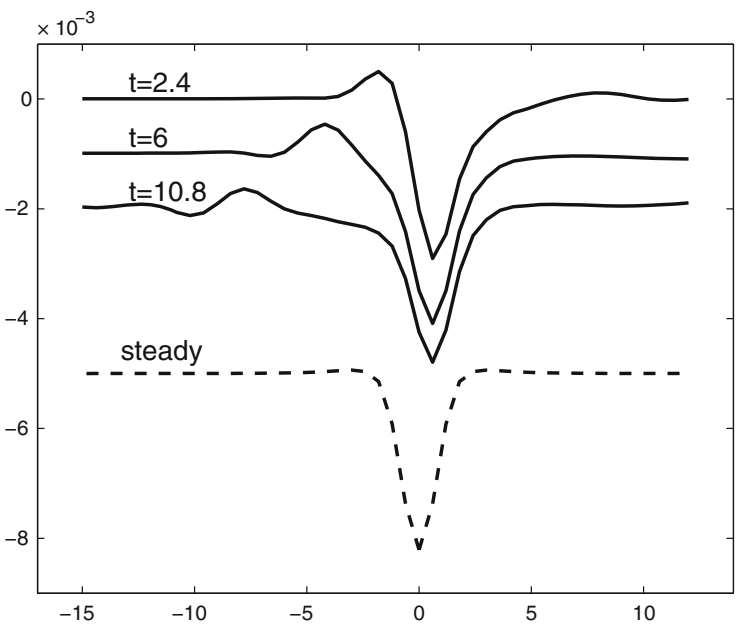

in front of the pressure distribution are seen to detach slower as $F$ increases. Their amplitude is increasing with $F$. A similar analysis can be performed by keeping $F$ constant and varying $\beta$.

We now turn our attention to the case of weak surface tension (i.e., $\alpha<1 / 4$ ). We can then expect to find waves on the free surface since the velocity $U$ at which the pressure distribution moves is greater than the minimum phase velocity of linear waves.

A linear analysis of the wave pattern was developed by Yih and Zhu [20] and Chen [21]. It is found that there is a set of capillary waves ahead of the moving pressure and two different sets of waves behind the moving pressure. One set of waves behind the moving pressure corresponds to the traditional Kelvin wake with transverse waves and divergent waves. These waves are mostly dominated by gravity, but they are also affected by capillarity. They have much larger wavelengths than the waves in front of the pressure distribution, so it is very difficult to capture both sets of waves with a uniform grid. The other set of waves behind the pressure distribution is mostly dominated by the capillarity. As the velocity of the moving pressure approaches the minimum phase velocity of linear waves, the divergent waves from the Kelvin pattern disappear. The angle of the region within which the transverse waves behind the pressure are confined increases from Kelvin's angle, classically $38^{\circ} 56^{\prime}$, and tends to $180^{\circ}$.

We observed this variation of the angle behind the moving pressure and the influence of surface tension on the wavelength of the waves behind the pressure (see Figs. 8, 9). In these figures, we kept $F=0.7$ and we increased 


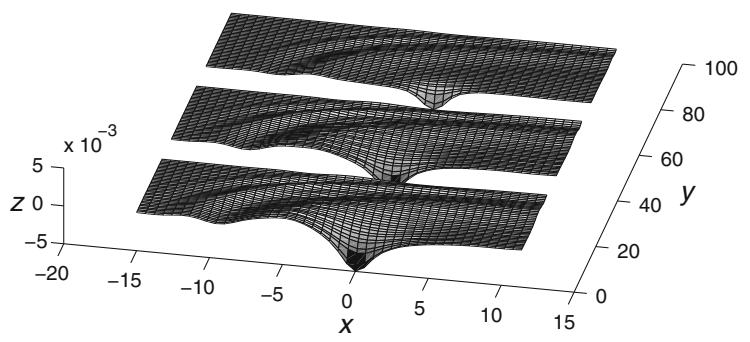

Fig. 6 Time-dependent forced gravity-capillary waves at a fixed time $(t=10.8)$ for $\beta=2$ and various $F: 1,1.5$ and 2 (top/middle/bottom). Only half of the solutions $(y>0)$ are plotted

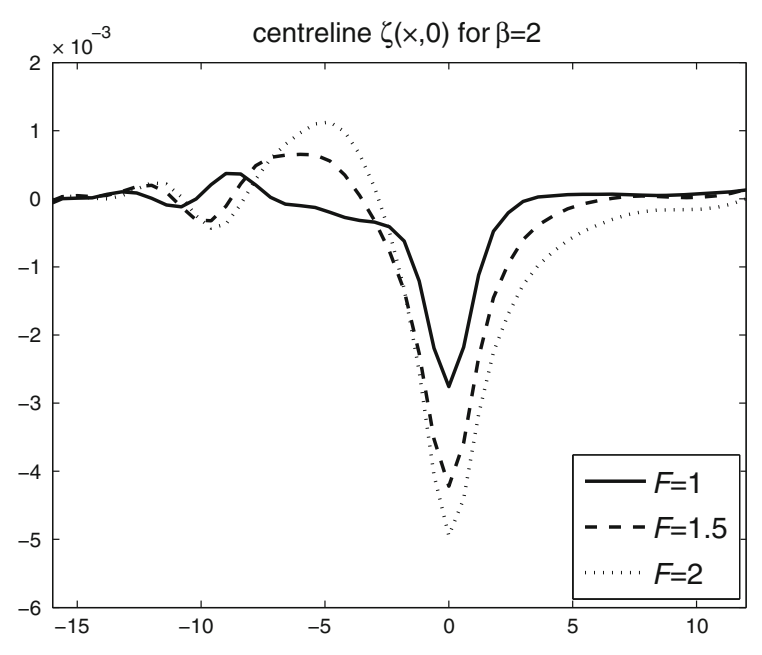

Fig. 7 Centreline $\zeta(x, 0)$ of the time-dependent forced gravity-capillary waves at a fixed time $(t=10.8)$ for different values of $F$, where $\beta=2$

$\beta$ from 0 to 0.1 . In Fig. 8 the three-dimensional solutions are plotted, after a long time has passed so that they are essentially steady. In Fig. 9 the transverse cuts of the solutions at a fixed location behind the pressure are presented. In Fig. 10, where the centrelines in $x$-direction of the solutions are plotted, the variation of the wavelength of the transverse waves with $\beta$ can be observed and it is found that it decreases as $\beta$ increases.

We also compare in Figs. 11-14 the steady gravity-capillary solutions obtained by Părău et al. [6] using the Rayleigh viscosity $\mu$ with an unsteady solution computed with the present algorithm. In this case the parameters are $F=\sqrt{3}$ and $\beta=0.6$, so we are close to the minimum phase velocity since $\alpha=0.2$, and we are in the strong-surface-tension regime. In these computations the pressure distribution is chosen as

$P(x, y)= \begin{cases}\varepsilon \mathrm{e}^{\frac{25}{x^{2}-25}+\frac{25}{y^{2}-25}}, & |x|<5 \text { and }|y|<5, \\ 0, & \text { otherwise, }\end{cases}$

where $\varepsilon=0.1$, in order to compare the unsteady solutions with the previously computed steady solutions.

We observe the capillary waves forming and developing in front of the pressure distribution. In the upper half of Fig. 11 the artificial viscosity in the steady problem is relatively high $(\mu=0.1)$, while in the upper half of the Fig. 12 it is decreased to $\mu=0.05$. In both figures the lower half show the unsteady solution at $t=72$. We can observe that the agreement between the steady and unsteady solutions improves as $\mu$ decreases.

This can also be observed in Fig. 13 (for $\mu=0.1$ ) and Fig. 14 (for $\mu=0.05$ ), where only the centreline of the solutions in the $x$-direction is presented. The solid line is the centreline of the solution obtained with the unsteady algorithm, while in both cases the dashed line represent the solution obtained with the steady algorithm from [6]. The agreement between the solutions is very good under the support of the pressure.

Our findings show that there are two ways to compute steady gravity-capillary solutions when $\alpha<1 / 4$. The first is to solve directly the steady equations by iterations (see [6]). It is then necessary to introduce a small dissipation $\mu$ to satisfy the radiation condition. This gives good results but with a damping of the waves. The alternative approach presented in this paper is to solve the time-dependent problem and to find numerically the long time behaviour. The advantage is that there is no damping of the waves. 
$\beta=0$.

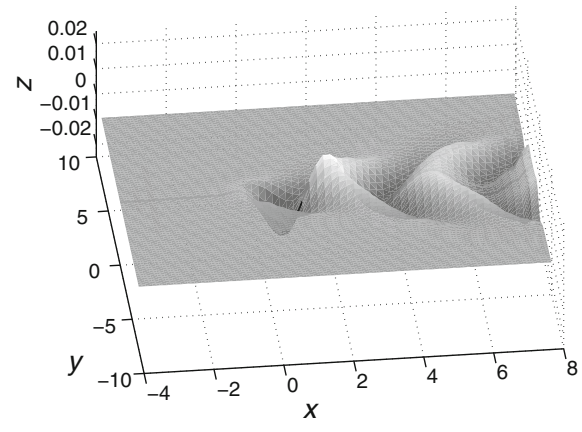

$\beta=0.02$

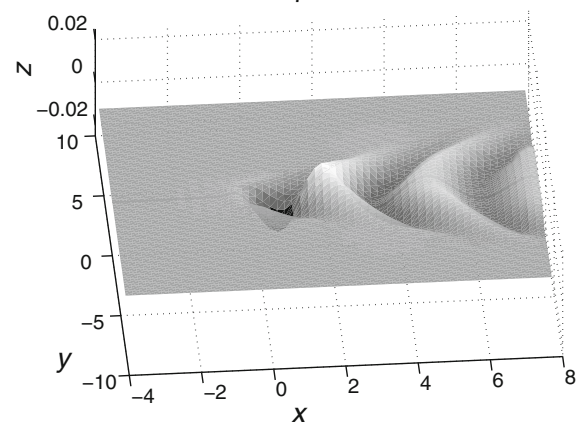

$\beta=0.05$

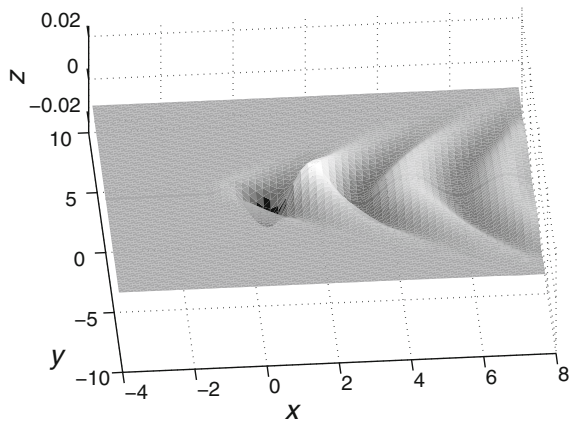

$\beta=0.1$

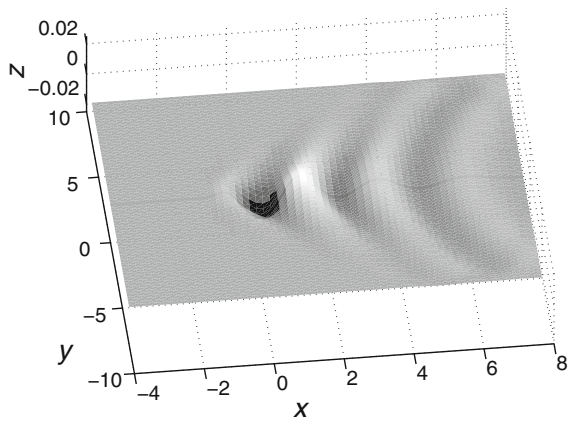

Fig. 8 Surfaces of time-dependent forced gravity-capillary waves at a fixed time $(t=24)$ for $F=0.7$ and various $\beta$. In these computations we have used $n=60, m=40, \delta x=\delta y=0.2, \delta t=0.05$. As $\beta$ increases no divergent waves are observed. The waves behind the pressure are no longer confined within the Kelvin angle of $38^{\circ} 56^{\prime}$

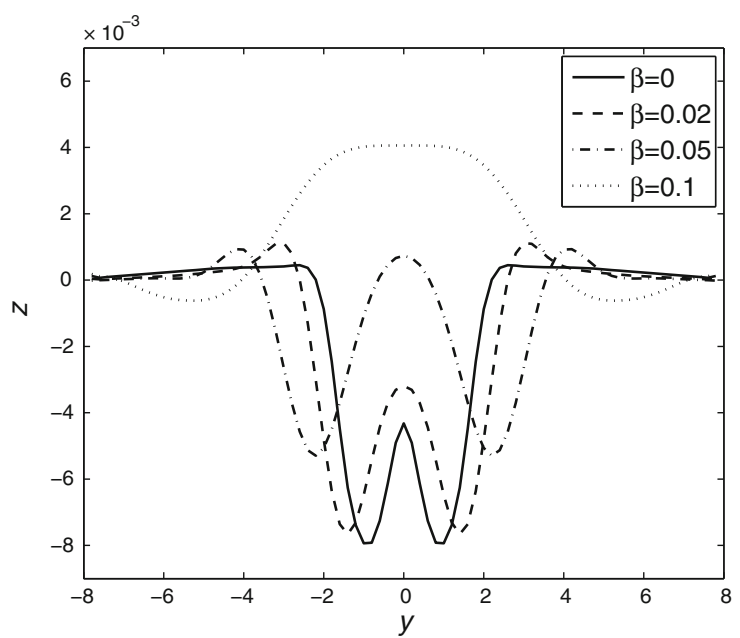

Fig. 9 As Fig. 8. Transverse cuts at $x=3.8$ in the $y$-direction of time-dependent forced gravity-capillary waves at a fixed time $(t=24)$ for $F=0.7$ and various $\beta$. The waves behind the pressure are no longer confined within the Kelvin angle $38^{\circ} 56^{\prime}$



Fig. 10 Centrelines in $x$-direction of time-dependent forced gravity-capillary waves at a fixed time $(t=24)$ for $F=0.7$ and various $\beta$. The wavelength of the transverse waves behind the pressure is decreasing as $\beta$ increases 


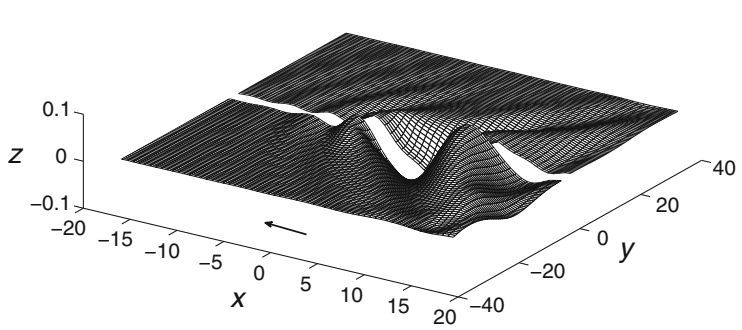

Fig. 11 Time-dependent and steady forced gravity-capillary waves for $F=\sqrt{3}, \beta=0.6$. In the upper part of the figure $(y>0)$ the steady solution is shown with $\mu=0.1$, while in the lower part $(y<0)$ the unsteady solution is shown at $t=72$

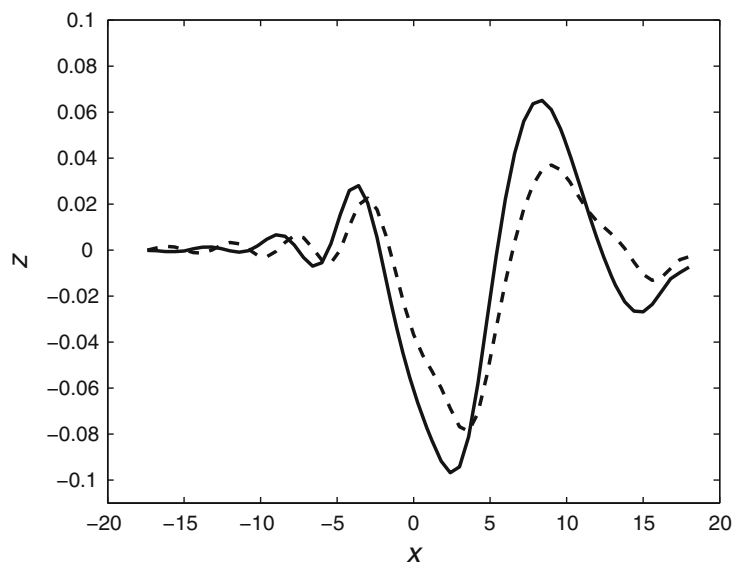

Fig. 13 Centreline $z=\zeta(x, 0)$ of the unsteady gravity-capillary waves at $t=72$ (solid line) and steady gravity-capillary waves (dashed line) for $F=\sqrt{3}, \beta=0.6$. The Rayleigh viscosity is $\mu=0.1$

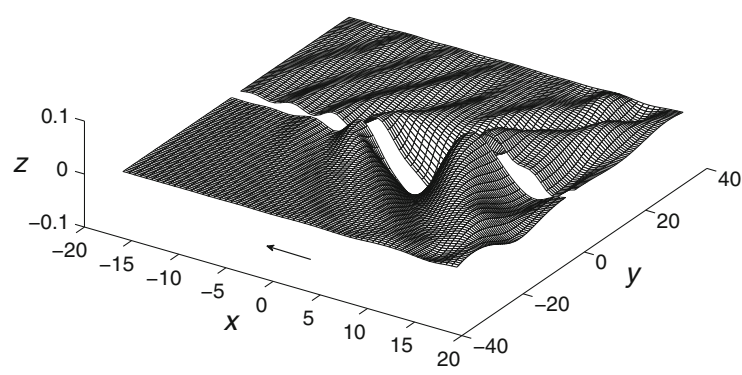

Fig. 12 Time-dependent and steady forced gravity-capillary waves in infinite depth for $F=\sqrt{3}, \beta=0.6$. In the upper part of the figure $(y>0)$ the steady solution with $\mu=0.05$ is shown, while in the lower part $(y<0)$ the unsteady solution at $t=72$ is shown

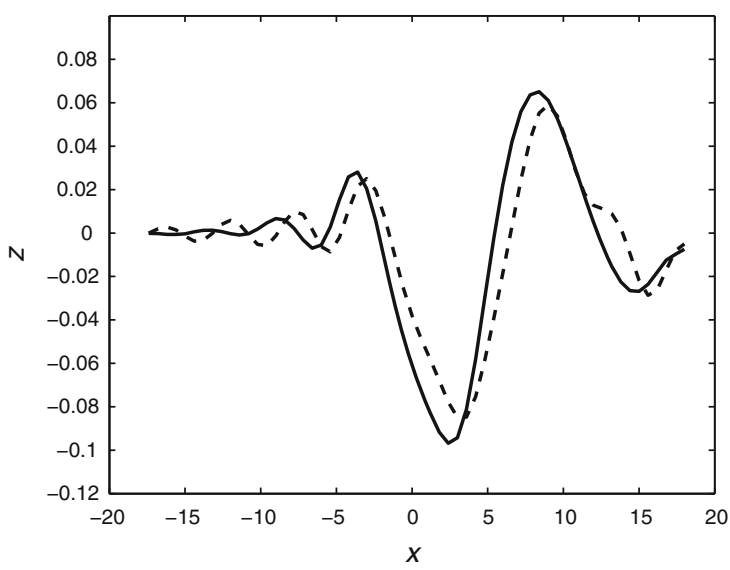

Fig. 14 Centreline $\zeta(x, 0)$ of the unsteady gravity-capillary waves at $t=72$ (solid line) and steady gravity-capillary waves (dashed line) for $F=\sqrt{3}, \beta=0.6$. The Rayleigh viscosity is $\mu=0.05$

\section{Conclusion}

We have presented a time-dependent method to study the fully-nonlinear unsteady gravity-capillary free-surface flows. The algorithm uses a boundary-integral method coupled with marching in time. Free-surface flows generated by a moving disturbance have been computed. Both gravity and surface tension were introduced in the dynamic boundary condition. Steady solutions satisfying the radiation condition have been obtained as the long-time behaviour of unsteady flows. In particular, we recovered for strong surface tension the steady solutions computed by Părău et al. [5]. We also recovered steady solutions for weak surface tension. These solutions had been calculated before by Părău et al. [6]. However, these previous calculations used a Rayleigh viscosity to satisfy the radiation condition. The present solutions were obtained without introducing any dissipation. In future we plan to investigate the stability of fully localised solitary waves solutions; see [4]. We also plan to generalise the algorithm to calculate time-dependent interfacial three-dimensional gravity-capillary flows.

Acknowledgement The research presented in this paper was carried out on the High Performance Computing Cluster supported by the Research Computing Service at the University of East Anglia. 


\section{References}

1. Părău EI, Vanden-Broeck J-M (2002) Nonlinear two- and three-dimensional free surface flows due to moving disturbances. Eur J Mech B/Fluids 21:643-656

2. Lord Rayleigh (1883) The form of standing waves on the surface of running water. Proc Lond Math Soc 15:69-78

3. Forbes LK (1989) An algorithm for 3-dimensional free-surface problems in hydrodynamics. J Comput Phys 82:330-347

4. Părău EI, Vanden-Broeck J-M, Cooker MJ (2005) Nonlinear three-dimensional gravity-capillary solitary waves. J Fluid Mech 536:99-105

5. Părău EI, Vanden-Broeck J-M, Cooker MJ (2005) Three-dimensional gravity-capillary solitary waves in water of finite depth and related problems. Phys Fluids 17:122101

6. Părău EI, Vanden-Broeck J-M, Cooker MJ (2007) Three-dimensional capillary-gravity waves generated by a moving disturbance. Phys Fluids 19:082102

7. Părău EI, Vanden-Broeck J-M, Cooker MJ (2007) Three-dimensional gravity and gravity-capillary interfacial flows. Math Comput Simul 74:105-112

8. Părău EI, Vanden-Broeck J-M, Cooker MJ (2007) Nonlinear three-dimensional interfacial flows with a fee surface. J Fluid Mech 591:481-494

9. Forbes LK (1983) Free-surface flow over a semicircular obstruction, including the influence of gravity and surface tension. J Fluid Mech 127:283-297

10. Grandison S, Vanden-Broeck J-M (2006) Truncation approximations or gravity-capillary free-surface flows. J Eng Math 54:89-97

11. Dias F, Bridges T (2006) The numerical computation of freely propagating time-dependent irrotational water waves. Fluid Dyn Res 38:803-830

12. Landweber L, Macagno M (1969) Irrotational flow about ship forms. Iowa Institute of Hydraulic Research Report-IIHR, vol 123, pp 1-33

13. Beck RF (1999) Fully nonlinear water wave computations using a desingularized Euler-Lagrange time domain approach. In: Mahrenholtz O, Markiewicz M (eds) Nonlinear water wave interaction advances in fluid mechanics series. WIT Press, UK pp 1-58

14. Sung HG, Grilli ST (2006) Combined Eulerian-Lagrangian or pseudo-Lagrangian descriptions of waves caused by an advancing free surface disturbance. In: Proceedings of the 16th international offshore and polar engineering conference, pp 487-494

15. Longuet-Higgins MS, Cokelet ED (1976) The deformation of steep surface waves on water. I. A numerical method of computation. Proc R Soc Lond A 350:1-26

16. Cao Y, Beck RF, Schultz WW (1993) An absorbing beach for numerical simulations of nonlinear waves in a wave tank. In: 8th international workshop on water waves and floating bodies, St. John's, Canada, pp 17-20

17. Vanden-Broeck J-M, Dias F (1992) Gravity-capillary solitary waves in water of infinite depth and related free-surface flows. J Fluid Mech 240:549-557

18. Dias F, Kharif C (1999) Nonlinear gravity and capillary-gravity waves. Annu Rev Fluid Mech 31:301-346

19. Lamb H (1932) Hydrodynamics, 5th edn. Cambridge University Press, Cambridge

20. Yih CS, Zhu S (1989) Patterns of ship waves II: gravity-capillary waves. Q Appl Math 47:35-44

21. Chen XB (2005) Analytical features of unsteady ship waves. In: Chwang AT, Teng MH, Valentine DT (eds) Advances in engineering mechanics_-reflections and outlook. Proceedings of the Theodore Y.T. Wu symposium, Vancouver, Canada, June 2004. World Scientific Pub. Co., River Edge, pp 371-389 